\title{
Multifaceted roles of tunneling nanotubes in intercellular communication
}

\author{
Ludovica Marzo ${ }^{1,2 \dagger}$, Karine Gousset ${ }^{1 \dagger}$ and Chiara Zurzolo ${ }^{1,2 *}$ \\ ${ }^{1}$ Unité de traffic membranaire et pathogenèse, Institut Pasteur, Paris, France \\ ${ }^{2}$ Dipartimento di Biologia e Patologia Cellulare e Molecolare, Università Federico II, Napoli, Italy
}

Edited by:

Claudia Verderio, Consiglio Nazionale delle Ricerche, Italy

Reviewed by:

Hans-Hermann Gerdes, University of Bergen, Norway

Yan Zhang, Peking University, China

*Correspondence:

Chiara Zurzolo, Unité de traffic membranaire et pathogenèse, Institut

Pasteur, 25 rue du docteur Roux, 75074 Paris Cedex 15, France.

e-mail: chiara.zurzolo@pasteur.fr

${ }^{+}$Ludovica Marzo and Karine Gousset have contributed equally to this work.
Cell-to-cell communication and exchange of materials are vital processes in multicellular organisms during cell development, cell repair, and cell survival. In neuronal and immunological cells, intercellular transmission between neighboring cells occurs via different complex junctions or synapses. Recently, long distance intercellular connections in mammalian cells called tunneling nanotubes (TNTs) have been described. These structures have been found in numerous cell types and shown to transfer signals and cytosolic materials between distant cells, suggesting that they might play a prominent role in intercellular trafficking. However, these cellular connections are very heterogeneous in both structure and function, giving rise to more questions than answers as to their nature and role as intercellular conduits. To better understand and characterize the functions of TNTs, we have highlighted here the latest discoveries regarding the formation, structure, and role of TNTs in cell-to-cell spreading of various signals and materials. We first gathered information regarding their formation with an emphasis on the triggering mechanisms observed, such as stress and potentially important proteins and/or signaling pathways. We then describe the various types of transfer mechanisms, in relation to signals and cargoes that have been shown recently to take advantage of these structures for intercellular transfer. Because a number of pathogens were shown to use these membrane bridges to spread between cells we also draw attention to specific studies that point toward a role forTNTs in pathogen spreading. In particular we discuss the possible role that TNTs might play in prion spreading, and speculate on their role in neurological diseases in general.

Keywords: tunneling nanotubes, intercellular communication, long-range connections, vesicular transport, signal spreading, pathogen spreading, organelle transfer

\section{INTRODUCTION}

The ability of cells to communicate with each other is essential for the life of a multicellular organism and is evolutionarily conserved between species (Gurke et al., 2008). Without cell-to-cell communication, processes such as remodeling of tissues and organs, differentiation during development, growth, cell division, and responses to stimuli could not take place. Therefore, a great number of cellular genes and their products are implicated in intercellular communication and their misregulation leads to the establishment of pathological conditions associated with many diseases.

Chemical signaling by secretion of small molecules toward distant cells is the classical form of cell-to-cell communication and does not involve physical contact. It includes chemical mediators with paracrine effects on cells nearby, release of synaptic vesicles containing neurotransmitters between neurons (chemical synapses; Süudhof, 2008), and hormones, which travel in the blood stream after their release and can reach and stimulate distant target cells.

In cases of close proximity, cells can interact with each other through gap junctions or synapses. Gap junctions connect the cytoplasm of two neighboring cells by clustering tens to thousands of intercellular channels, allowing the transfer of ions and small, hydrosoluble molecules (Maeda and Tsukihara, 2011).
They mediate electrical and metabolic coupling of cells and are implicated in a wide range of biological processes such as muscle contraction or electrical synapses in neurons (Connors and Long, 2004). Immunological synapses, established at the interface between a T-cell and an antigen-presenting cell (APC), are rather mediated by membrane receptors (Rechavi et al., 2007; Tarakanov and Goncharova, 2009) and are essential for the adaptive immune response (Dustin et al., 2010). Structurally similar to the immunological synapse are the virological synapses. These supramolecular structures are cytoskeleton-dependent adhesive junctions induced by virus-infected cells and used by these pathogens to directly transfer to non-infected cells (Jolly and Sattentau, 2004). Human immunodeficiency virus type 1 (HIV-1) and human Tcell leukemia virus type 1 (HTLV-1) can spread using virological synapses between T cells (Tarakanov and Goncharova, 2009).

Recently, long-range forms of intercellular communication consisting of different types of membrane bridges have been described in a wide variety of cell types in in vitro cell culture systems (Gerdes et al., 2007; Abounit and Zurzolo, 2012; Figure 1). Similar connections have also been found in vivo and in tissue explants (Wolpert and Gustafson, 1961; Miller et al., 1995; Ramírez-Weber and Kornberg, 1999; Demontis and Dahmann, 2007; Chinnery et al., 2008). The discovery of these new types of 


\section{Non-mammalian long intercellular communications}
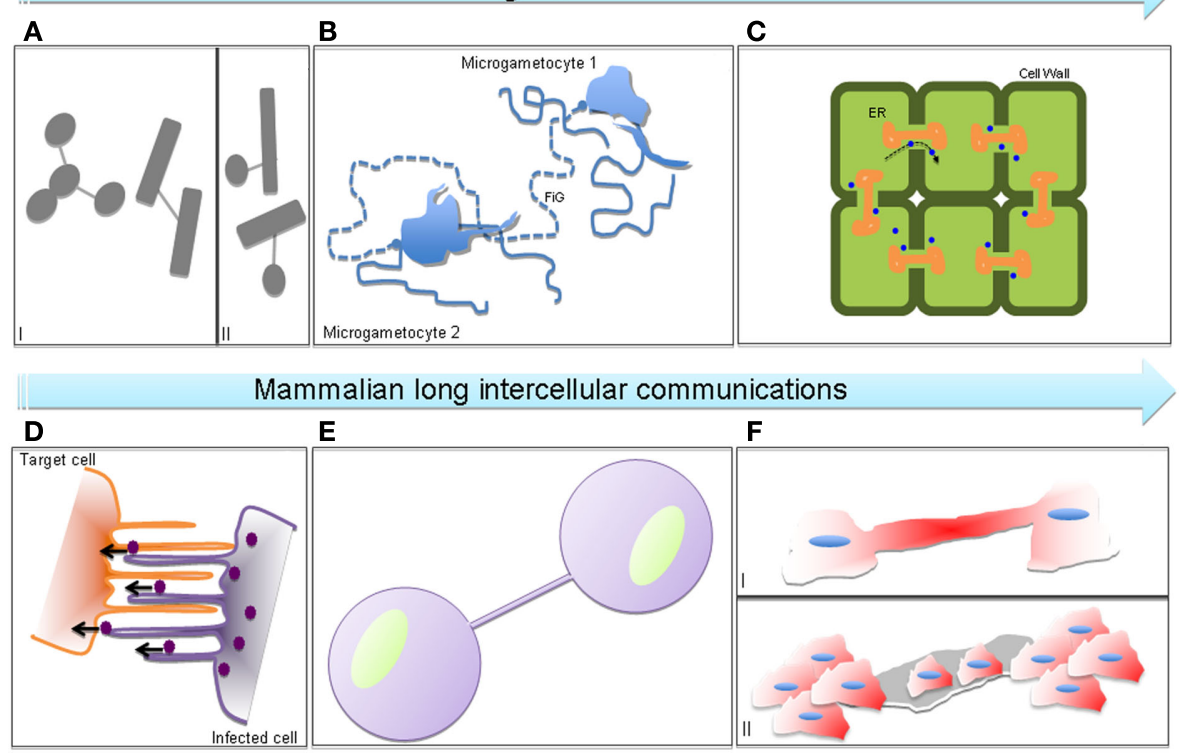

FIGURE 1 | Schematic representation of non-mammalian and mammalian long intercellular communications. (A) Nanotubes formed between bacteria of the same (I) and distinct (II) species, (B) filamentous connection (FiG) between extra flagellating microgametocytes (malaria sexual stage parasites), (C) plasmodesmata connecting neighboring plant cells, constituted by a membrane-lined cytoplasmic channel traversing the cell wall with an endoplasmic reticulum (ER) tubule passing through the middle and allowing the passage of molecules (blue dots), (D) viruses (violet dots) spreading on filopodial bridges or inside viral cytonemes formed between virus-infected and target cells, (E) tunneling nanotubes connecting mammalian cells, (F) type I (I) EP (epithelial) bridge connecting human bronchial EPs and type II EP bridge (II) formed between two EP islands of human bronchial EPs and allowing the passage of entire cells. communication highways has opened up new ways of viewing how cells interact with one another, leading to the reconsideration of the traditional view of the cell as a basic unit of structure, function, and organization originally postulated by Schwann and Schleyden (1847).

Tunneling nanotubes (TNTs; Figure 1E) were initially described by Rustom et al. (2004) as long thin actin-containing bridges connecting PC12 cells in culture that do not contact the substratum, extending up to $100 \mu \mathrm{m}$ in length with diameters ranging from 50 to $200 \mathrm{~nm}$. Since then, TNTs have been found in many cell types in culture, from immune to neuronal cells and primary cells, acting as conduits for cytosolic and membrane-bound molecules, organelles and spreading of pathogens (Gerdes et al., 2007; Gousset and Zurzolo, 2009).

Filopodial bridges (Figure 1D), also called viral cytonemes for their similarity with cytonemes (e.g., filopodial protrusions described in Drosophila imaginal disks; Ramírez-Weber and Kornberg, 1999), are instead cellular extensions observed in different cell types (Cos-1, HEK293, DFJ8, XC cells) and induced by some retroviruses before their entry into the cell (Sherer et al., 2007). It has been shown that murine leukemia virus (MLV) and HIV-1 can be unidirectionally transported on the surface of these structures, using them for cell-cell transmission and spreading (Lehmann et al., 2005; Sherer et al., 2007; Mothes et al., 2010). Vesicular clusters containing VP16, a structural protein of herpes simplex virus (HSV), and US3 kinase of the pseudorabies virus have been found in similar cellular projections, mainly at the contact site with neighboring cells, respectively in Vero cells (La Boissière et al., 2004) and RK13 cells (Favoreel et al., 2005).

In addition, Zani et al. (2010) have described two different types of cellular bridges (Figures 1FI,II), called epithelial bridges (EP bridges) that connect primary human bronchial epithelial cells. Differently from TNTs, EP bridges are more stable, longer (from $25 \mu \mathrm{m}$ up to $1 \mathrm{~mm}$ ), and with a diameter ranging from 1 to $20 \mu \mathrm{m}$ (Zani and Edelman, 2010). Structurally, they contain both F-actin and microtubules, similar to the TNTs found in primary human macrophages (Onfelt et al., 2006), rat cardiac myoblast cells (He et al., 2010), and cardiomyocytes/cardiofibroblasts co-culture system (He et al., 2011). While type I EP bridges (Figure 1FI) seamlessly allow the bi-directional transfer of different cellular components (e.g., lysosomes and Golgi), the type II structures (Figure 1FI) might represent a new way of cell migration since it can transfer an entire cell from one multicellular EP island to another (Zani and Edelman, 2010).

The discovery of mammalian bridges is more recent compared to plant conduits, called plasmodesmata (PDs; Figure 1C), because they are more fragile and more difficult to observe. For example, they are sensitive to prolonged light excitation, mechanical stress, and chemical fixation and are close to the optical limit of resolution (Hurtig et al., 2010). PDs share some structural characteristics with TNTs. They are thin membrane structures with a diameter around $50 \mathrm{~nm}$ but are shorter than TNTs as their length is determined by the thickness of the cell walls between neighboring cells. Moreover, PDs allow an actin-mediated transfer of small 
molecules, transcription factors, and also spreading of viruses, creating a sort of continuity between the cytoplasm of connected cells (symplast; Lucas et al., 2009). Even more similarities between the mammalian TNTs and the plant PDs are found regarding the mechanisms of formation and transfer (e.g., passive diffusion of small molecules and gated-mechanisms for bigger components) although the nature of the transported molecules can vary (Rustom, 2009; Abounit and Zurzolo, 2012). This highlights a possible common origin during evolution of TNTs and PDs that can allow a better understanding of the newly discovered mammalian bridges by comparing them with the better-known PDs.

Interestingly, along the same line of thinking, recent findings of bacterial networks (Figure 1A) and parasite protrusions (Figure 1B) make us wonder how evolutionally conserved these kinds of intercellular communications can be. Indeed, Dubey and Ben-Yehuda (2011) have recently shown that Bacillus subtilis grown on a solid surface can establish nanotube-mediated networks with neighboring bacteria of the same or different species (Figures 1AI,II), as Staphylococcus aureus or Escherichia coli, pointing toward a common way of communication shared between phylogenetically distant bacterial species. These linking structures and their mammalian or plant counterparts facilitate transfer of cytoplasmic components and non-conjugative plasmids, allowing the exchange of hereditary traits for the acquisition of new features between connected bacteria (Dubey and BenYehuda, 2011). Sometimes in nature similarities in structure do not reflect related functions. This could be the case of the cell-to-cell connections formed by the malaria pathogen during reproduction in the mosquito midgut (Rupp et al., 2011; Figure 1B). In this paper, the authors have described the presence of filamentous structures containing F-actin, that they called "filaments of gametes" or FiGs, in the activated gametocytes. Multiple FiGs are generated on the surface of the cell a few minutes after activation and can extend up to $180 \mu \mathrm{m}$. A closer look at these structures revealed that they possess closed-ends and they do not transfer material. Interestingly, FiGs exhibit adhesion molecules on their surface that can instead mediate contact and recognition with the right mating partners for the Plasmodium, allowing clustering of gametocytes and facilitating the process of reproduction (Rupp et al., 2011).

This and the other examples of intercellular contacts established by different types of cells reported here reveal a high heterogeneity in both structure and functions of these fascinating new routes of communication that need further characterization and classification (Figure 1). Furthermore in order to better understand their physiological relevance more efforts will be needed to identify these structures in vivo. To this aim the identification of specific TNT markers by using in vitro models is of fundamental importance.

This review will focus on mammalian TNTs, their possible mechanism of formation and their various functions, giving particular attention to their implication in prion spreading.

\section{MECHANISMS OF TNT FORMATION AND PROTEINS INVOLVED}

In two-dimensional cultures, TNT-like structures were first discriminated from filopodia from their structural space. Contrary to filopodia, they formed long bridges between cells and were not attached to the substratum (Rustom et al., 2004). In addition to their spatial differences, TNTs, and filopodia appear to serve different purposes. While filopodia act as important environmental sensors and play key roles in cell motility, the main role of TNTs appears to be as a direct conduit for cell-to-cell communication, specifically in the transport of material from one cell to another. As stated above, numerous membrane bridges have been described in a multitude of cell types. Even within TNT-like structures, it became quickly evident that these various structures were distinct from one another both in their structures and functions.

\section{TNT FORMATION AND STRUCTURAL COMPONENTS}

Tunneling nanotube-like structures were first described in PC12 neuronal cells (Rustom et al., 2004). In these cells, de novo actindriven formation of TNTs was observed. Further examination of PC12 cells and TNT formation suggested that while the majority of tubes formed via directed filopodia-like protrusions, a small subset $(7 \%)$ were also able to form after cells previously in contact detached from one another (Bukoreshtliev et al., 2009) (for review, see Abounit and Zurzolo, 2012). In the mouse neuronal CAD cell line, we were also able to observe both types of TNT formation (data not shown). However, the significance and the differences between these two modes of formation and whether they lead to various structures remain unclear. Similar to other cell types, we observed a high degree of heterogeneity in the diameters of TNT-like structures (Gousset et al., 2009). Furthermore, as previously described in PC12 cells (Rustom et al., 2004), neuronal TNTs formed between CAD cells contained actin filaments but no microtubules, even in the tubes with larger diameters (Gousset et al., 2009). The fact that most TNTs in neuronal cells arise from the extension of filopodia-like protrusions toward neighboring cells suggested that actin polymerization plays an important role in this type of TNT formation. Rustom et al. (2004) demonstrated that using the F-actin depolymerizing drug latrunculin, no TNTs were detected in PC12 treated cells. This type of treatment could thus be used to selectively block TNT formation and look at the effect of the presence or absence of nanotubes in various cultures. In our lab, we took advantage of this treatment to highlight the importance of the presence of TNTs in the transfer of infectious prion aggregates in neuronal cells (Gousset et al., 2009). Using nanomolar concentrations of Cytochalasin D (CytoD), another actin-depolymerizing drug, Bukoreshtliev et al. (2009) went further and examined the effects of this drug during the lifetime of TNTs. They showed that as expected, low levels of CytoD abrogated both filopodia formation and TNT formation. Interestingly, they also demonstrated that once formed, CytoD had little effects on the stability of these tubes or their ability to transfer material from one cell to another. Thus, most neuronal TNTs arise from filopodia-like structures, detached from the substratum. Once formed however, they are no longer sensitive to low levels of actindepolymerizing drugs, demonstrating that functional TNTs are distinct from filopodia in both structure and function. Interestingly, recent experiments with primary rat astrocytes and neurons also showed actin to be the major cytoskeleton component of TNTs formed between these cells (Wang et al., 2011). Indeed, these 
authors showed that treatment with latrunculin or CytoD abrogated their formation, thus further validating the use of neuronal cell lines as models for neuronal TNTs.

Tunneling nanotube-like structures have also been described in immune cells, such as B-cells, Natural killer cells, and macrophages (Onfelt et al., 2004). In macrophages, two types of nanotubes were described (Onfelt et al., 2006). The thin nanotubes were found to contain actin filament only, whereas thicker nanotubes, with diameters larger than $0.7 \mu \mathrm{m}$, contained both F-actin and microtubules. These different structures appeared to have distinct functions, with the thicker structures being able to transport in a bi-directional manner vesicles and various organelles in a microtubule dependent mechanism. Similarly, long nanotube connections between Jurkat $\mathrm{T}$ cells and primary $\mathrm{T}$ cells were also described (Sowinski et al., 2008). In these cells, F-actin but no microtubules were detected in TNTs. In addition, while these tubes were not open-ended, they still allowed for the transfer of HIV-1 via a receptor-dependent mechanism. Finally, numerous networks of TNT-like structures were observed between dendritic cells and THP-1 monocytes (Watkins and Salter, 2005). These connections varied greatly in length and diameter but were able to quickly transfer calcium fluxes and small dyes to interconnected cells.

Thus, while numerous TNT-like structures have been described in immune cells, these tubes are clearly distinct from one another both in their structural components as well as in their means of transfer. The one characteristic consistent for all types of immune cells is their formation that appears to rely primarily on cell-to-cell attachment and formation of immunological synapses prior to cell separation and tube formation (Sowinski et al., 2008).

In urothelial cell lines, two types of TNT-like structures were observed (Veranic et al., 2008). The shorter but more dynamic structures, described as Type I nanotubes, were found to contain actin and to connect with neighboring cells by an anchoring type of intercellular junctions. By using time-lapse phase-contrast microscopy the authors observed that these structures did not collapse after micromolar concentrations of CytoD suggesting that after anchoring actin was no longer necessary (Veranic et al., 2008). On the other hand, the longer and more stable structures, or type II nanotubes, no longer contain actin filaments but were composed of cytokeratin filaments. Although the authors have observed vesicles on both these types of structures, further investigation is necessary to understand if these structures are involved in transferring materials, thus fulfilling the TNT definition.

These examples show the disparity in the various cytoskeleton requirements and formation mechanisms in naturally occurring TNT-like structures in neuronal, immunological, or epithelial cells. The type of formation however (de novo actin-driven vs detachment after cell-to-cell contact) might arise from the nature and role that these cells play in vivo. Indeed, mobile cells, which can more easily come into contact with other cells, might be more prone to form tubes from a previous cell-to-cell contact, whereas more immobile cells might be more adept at creating and extending tubes de novo toward distant cells. Because of the increasing number of studies on different and highly heterogeneous TNT-like structures in several in vitro systems a more systematic classification is needed.

\section{SIGNALS AND MOLECULES INVOLVED IN TNT FORMATION: IS STRESS A MAJOR PLAYER?}

In order to better understand the role that TNTs may play in intracellular transfer of materials, a better characterization of the initiation steps of TNT formation, the signals that guide the extension of these structures toward a neighboring cell and the mechanisms of binding and fusion need to be elucidated.

Recently, the effects of stress on TNT formation have been analyzed in different cell types (Wang et al., 2011). In their studies, Wang and colleagues have shown that stress induced by hydrogen peroxide $\left(\mathrm{H}_{2} \mathrm{O}_{2}\right)$ treatment led to an increase in TNT formation in both astrocytes and neurons. They also observed the transfer of various organelles, such as ER, Golgi, endosomes, and mitochondria via TNTs in astrocyte cultures. For both astrocytes and neurons, it was always the cells undergoing stress that developed TNTs and transferred cellular materials in a unidirectional fashion to the non-activated cells, suggesting that TNT formation might be directly induced by stress and may represent a defense mechanism of the stressed cells. Interestingly, they found that p53 activation, which is critical in apoptosis, led to an increase in TNT formation. Conversely, down regulation of p53 blocked TNT formation (Wang et al., 2011). Subsequently, they showed that EGF receptor up-regulation was also necessary for TNT initiation using different conditioned media and that the initiation of TNT formation was likely dependent on the initiating cells and not the receiving cells. Finally, since the EGF receptor can activate the Akt/PI3K/mTOR pathway, they used various mutants and inhibitors to selectively block or activate each protein and found that this pathway was indeed up regulated in $\mathrm{H}_{2} \mathrm{O}_{2}$ activated cells, leading to an increase in TNT development (Wang et al., 2011). In another study, using a macrophage cell line and HeLa cells, it was demonstrated that the interaction between $\mathrm{m}$-Sec and the Ral/exocyst complex was also critical for TNT formation (Hase et al., 2009). Therefore, to understand if $\mathrm{m}$-Sec might also be important for TNT formation in astrocytes, Wang et al. (2011) analyzed by RT-PCR the levels of $\mathrm{m}$-Sec in astrocytes and found a positive relationship between $\mathrm{H}_{2} \mathrm{O}_{2}$ treatment and the levels of $\mathrm{m}-\mathrm{Sec}$ expression. Interestingly, their data indicated that $\mathrm{m}-\mathrm{Sec}$ might be regulated by $\mathrm{p} 53$ activation. Thus, the authors suggest that the initiating cells control TNT formation in a $\mathrm{p} 53$ and $\mathrm{Akt} / \mathrm{PI} 3 \mathrm{~K} / \mathrm{mTOR}$ pathway activationdependent manner, but they do not exclude that some guidance cues might be originating from the receptor cells (Wang et al., 2011). Further studies are required in order to explore other potential molecular targets downstream of p53 and Akt/PI3K/mTOR pathways that might represent key elements involved in TNT formation.

In another study Yasuda et al. (2010) analyzed the transfer of mitotracker labeled vesicles via TNTs between endothelial progenitor cells (EPC) and human umbilical vein endothelial cells (HUVEC). They observed both TNT formation between the twocell types and transfer of mitochondrial material from the EPC to the HUVEC. Upon treatment of the HUVEC with adriamycin, they observed a large increase in the transfer of mitotracker particles from the non-stressed EPC to the adriamycin-stressed HUVEC. In addition, the transfer was unidirectional since the reverse loading and transfer experiments were not significant (Yasuda et al., 2010). While it was not clear in these experiments 
which cell type initiated the formation of the nanotubes, contrary to what was found in neuronal and astrocyte cultures (Wang et al., 2011), the transfer of material occurred from the non-stressed cells to the stressed cells. These observations raised the question of how these cells initiated TNTs. Further characterization in these cocultures could determine whether the stressed cells might release some signals that might attract filopodia-like protrusions from the EPC to the HUVEC or whether the HUVEC cells might initiate formation and allow for a reverse transfer of material from the receptor cell to the initiator cell.

This is exactly what the authors next set out to demonstrate. Indeed, in a follow-up study, they analyzed more precisely the TNT formation mechanisms between these cells. First they showed that co-cultures of EPC with stressed HUVEC led to a rescue of HUVEC viability. However, when the EPC were pre-treated with nanomolar levels of CytoD to block TNT formation prior to coculture with the HUVEC, the rescue effects were almost entirely abrogated, pointing toward the importance of TNT formation from EPC to HUVEC for cell survival. Using both fluorescence microscopy and FACS analyses they observed basal levels of transfer of lysosomes between the two-cell types in a bi-directional manner under non-stressed conditions. However, the transfer was much more efficient as it increased in speed and frequency and was found preferentially between non-stressed EPC and GC-stressed HUVEC, suggesting that the stressed cells were able to signal and guide filopodia-like protrusions for the formation of de novo TNTs to occur (Gerdes et al., 2007; Yasuda et al., 2010, 2011). Further examination suggested that surface-exposed phosphatidylserines (PS) in HUVEC might be able to guide TNT formation from the EPC to the stressed HUVEC. Indeed, when PS on HUVEC were blocked by binding of Annexin V, the selective TNT formation and transfer from EPC to HUVEC was also blocked (Yasuda et al., 2011).

Overall, these studies suggest that transfer of materials via TNTs in most cell types occurred from the cell type that initiated TNT formation to the receptor cell. However, while certain stress conditions might increase the formation of TNTs between cells, it does not affect all cells the same way. Indeed, while in astrocytes and neurons, stress appears to increase TNT formation in the stressed cells leading to an increase in transfer of material, in endothelial cells stress increase the guidance signals from the stressed cells leading to an increased formation of TNTs from the nonstressed cells. Thus, once more the analysis of these two studies brings forward the disparities that exist in formation and nature of TNTs between different cell types. It suggests that even within an identical type of TNT formation (i.e., de novo extension of filopodia-like protrusions) the mechanisms might be very distinct from one another (activation of attractive guidance signals vs activation of initiation of filopodia-like protrusions). However, these studies implicate the involvement of more general signaling pathways in TNT formation. For example, the role of $\mathrm{m}$-Sec, which was found to be important in macrophages, HeLa cells, and astrocytes (Hase et al., 2009), could be of general importance in TNT formation, independent of cell type. In addition, since filopodialike protrusions are critical for TNT formation in neuronal cells (Bukoreshtliev et al., 2009), our lab, has turned its attention to the role that the actin molecular motor protein Myosin-X might play in both the formation of TNT-like structures and its function in transfer of materials in neuronal cells. We found that overexpression of Myosin-X (Berg and Cheney, 2002) increased the number of TNTs observed in our cell cultures (data not shown). In addition, similar to what Wang and colleagues (Wang et al., 2011) have found with stress signals, we observed a unidirectional transfer of vesicles occurring from the cells over-expressing Myo-X to the acceptor cells (data not shown).

Finally the search for guidance signals and the role that lipids might play in TNT formation might provide further information about TNT formation.

\section{MECHANISMS INVOLVED IN OPEN-ENDEDNESS OF TNTS}

As previously stated, in T cells no membrane continuity or transfer of cytosolic material have been observed (Sowinski et al., 2008), suggesting different types of tubular structures between $\mathrm{T}$ cells and other cell types that allowed for the transfer of cytosolic materials such as neuronal cells, astrocytes, myeloid cells, or endothelial cells. Recently, however, Arkwright et al. (2010) have shown that specific stimulation could lead to an increase of TNTs in T cells along with the transfer of cytosolic material. First, they showed that FAS activation resulted in an increase in TNT formation and that both toxin $\mathrm{B}$ of Clostridium difficile (an inhibitor of actin Rho-GTPases) and secramine A (an inhibitor of CDC42) specifically blocked FAS stimulated TNT formation in T cells. They also analyzed the bi-directional exchange of labeled membranes in T-cell co-cultures. As expected, they only found a negligible number of TNTs with both markers in control cells, whereas upon FAS stimulation they observed a 20 -fold increase in the number of TNTs labeled with both membrane markers. The transfer of cytosolic materials, including fluorescent cytosolic proteins as well as labeled vesicles, was also observed upon FAS-stimulation between $\mathrm{T}$ cells. These experiments demonstrated that the nanotubular structures initiated by FAS-stimulation were different from the TNTs previously described in non-activated T cells and did not contain an immunological synapse (Sowinski et al., 2008). These connections were similar to the connections observed in other cell types and demonstrate the complexity and dynamism of the various TNT-like structures that have been described to date. While this study demonstrates that within the same cells, different activation can quickly lead to the formation of different types of TNTs with distinct functions; the mechanisms involved in the gating of these tubular structures remain undetermined. Overall, these recent studies on TNTs have shown the diversity of these structures but also their ability to transfer numerous signals upon specific activation.

\section{CAUSE AND CONSEQUENCES OF TNT-MEDIATED TRANSFER, FROM SIGNAL TO ORGANELLES AND PATHOGENS}

Tunneling nanotubes have revealed a high degree of heterogeneity also from a functional point of view, as different components seems to be selectively transferred by different cell types. What determines this selectivity remains unknown.

First, further investigation is needed to understand why some cargoes are unidirectionally or bi-directionally transported. Unilateral transfer occurs in the case where a donor cell transfers material to an acceptor cell, whereas bi-lateral transfer happens 
when both cells mutually exchange materials. The reasons for these different transport mechanisms can depend on the structural components (actin only vs actin + microtubules containing TNTs) or on specific signals that stimulate nanotube formation and are responsible for directing the traffic in one or two ways.

As already mentioned above, bi-directional transfer is found when both actin and microtubules are present (Onfelt et al., 2006; Arkwright et al., 2010; He et al., 2010, 2011), while it appears to be unidirectional when TNTs contain actin only (Rustom et al., 2004; Koyanagi et al., 2005; Gurke et al., 2008; Eugenin et al., 2009; Gousset et al., 2009; Domhan et al., 2011). A recent work by Plotnikov et al. (2010) shows that unidirectional transfer from rat renal tubular cells (RTC) to bone marrow multipotent mesenchymal stromal cells (MMSC) was observed in this co-culture system (Plotnikov et al., 2010). However, passage of molecules in the opposite direction was also detected, albeit at a lower rate. Additionally, it has been shown that lysosome exchange (Lysotracker-labeled) between endothelial progenitor cells (EPC) and endothelial cells (HUVEC) in co-cultures occurs at a basal level and that this transfer selectively increases in one direction, from EPC to HUVEC cells, upon injury of the latter (Yasuda et al., 2010). These two reports suggest that a shift from a bi-directional basal level of transfer to a selective unidirectional transfer toward a specific cell population might take place by means of intercellular thin connections resembling TNTs between cells upon specific treatment, as is the case for differentiation signal flow toward MMSC cells (Plotnikov et al., 2010) and stress signal deriving from damaged organelles (Yasuda et al., 2010). What remains to be determined is how transfer occurs within TNTs and whether common molecular motors might be involved during this process. Furthermore, the fact that TNT structures contain F-actin as backbone suggests that an acto-myosin-dependent mechanism could be responsible for organelles or pathogens transfer mediated by TNTs (Rustom et al., 2004; Gerdes et al., 2007; Hurtig et al., 2010). It has been reported that organelle transfer through TNTs is an active process that depends on actin and ATP (Onfelt et al., 2006; Gurke et al., 2008; Bukoreshtliev et al., 2009; Gousset et al., 2009). Indeed the use of F-actin depolymerizing drugs and ATP-depletion experiments resulted in an almost complete block of organelle transfer (Onfelt et al., 2006; Gurke et al., 2008; Bukoreshtliev et al., 2009; Gousset et al., 2009). Furthermore by measuring the trajectory of the organelles transferring from one cell to another Gurke et al. (2008) demonstrated that the vesicle movement inside TNTs of NRK cells was due to active transport and not to free diffusion. Similar conclusions were obtained by measuring the mean square displacement of PrP containing vesicles in TNTs (Gousset et al., 2009). In addition, vesicular traffic on actin- and microtubulescontaining TNTs in macrophages was shown to be sensitive to ATP-depletion, indicating that independently of the cytoskeleton components transfer through TNTs occurs as an active process (Onfelt et al., 2006). Finally the actin-binding motor Myosin Va is present in TNTs and partially localizes with endocytic organelles (Rustom et al., 2004; Gerdes et al., 2007). A more detailed analysis on the role of Myosin Va and the screening of myosin motors involved in endocytic vesicles traffic or pathogens spreading will be necessary to further dissect the mechanism of transfer occurring via TNTs.

\section{SIGNAL TRANSFER}

Up to now several reports have shown that calcium signals could propagate between remote cells through TNTs (Watkins and Salter, 2005; Hase et al., 2009; Smith et al., 2011; Table 1). This is especially important for remote cells that are unable to propagate calcium-mediated signaling to cells in close proximity using gap junctions (Wang and Gerdes, 2011). Initially, Watkins and Salter (2005) demonstrated that myeloid cells can respond to stimulation through soluble factors or mechanical stress and are able to amplify the cellular response by calcium signaling through membrane connections. Since then, propagation of calcium flux has been shown in many other cell types able to make connections between each other (Hase et al., 2009; Smith et al., 2011; Table 1). More recently, the transfer of IP3 receptor (IP3R) and endoplasmic reticulum has been described along TNTs in SH-SY5Y neuroblastoma and HEK cell lines (Smith et al., 2011). The authors made a comparison between the current produced at the end of a TNT (typically $30 \mu \mathrm{m}$ in length and $200 \mathrm{~nm}$ in diameter) and single inositol trisphosphate receptor (IP3R)-channels. While the first produces a current $<1 \mathrm{fA}$, corresponding to calcium flux propagated from an activated cell, the opening of a single channel results in $\sim 100 \mathrm{fA}$. Considering that a single opened IP3R-channel generally fails to induce $\mathrm{Ca}^{2+}$ signaling, the passive diffusion of $\mathrm{Ca}^{2+}$ within TNTs appears quite inefficient. However, since IP3R is able to transfer along TNTs, it could overcome the limit of passive diffusion of calcium by amplifying calcium signaling within a population. Finally, a recent study has reported the formation of electrically coupled nanotubes that do not allow diffusion of calcium or IP3R, but are instead involved in the bi-directional spread of electrical current between distant cells through gap junctions (Wang et al., 2010). These type of TNTs are immuno-positive for connexin-43, at one end of the connection and allow the passage of electrical signals which in turn leads to the activation of low voltage gated channels that allow a local influx of calcium in the connected cell. Electrical coupling-competent TNTs, distinguished from those that do not possess gap junctions, have been found in different cell types and represent a selective way for transferring electrical signals compared to gap junctions coupling (Wang et al., 2010; Wang and Gerdes, 2011; Abounit and Zurzolo, 2012).

Overall, calcium spreading through nanotubes appears to be a good option for different types of cells to quickly spread calcium signals under physiological conditions, leading to fast responses in connected neighboring cells (for review see Abounit and Zurzolo, 2012; Table 1).

Particularly fascinating and newly discovered is the spreading of death signals by nanotubes occurring in Jurkat and primary T cells (Arkwright et al., 2010; Table 1). Fas-mediated signaling is important for peripheral deletion of activated $\mathrm{T}$ lymphocytes (Green et al., 2003). Mutations in the cytoplasmic domain of the Fas receptor are responsible for a rare genetic disease, the autoimmune lymphoproliferative syndrome (the type Ia ALPS; Martin et al., 1999). As stated previously, Arkwright et al. (2010) have shown that stimulation of the Fas receptor leads to an increase in the number of TNT-connected cells and this is critically dependent on Rho GTPase activation. Accordingly, the authors also demonstrated that primary $\mathrm{T}$ cells deriving from ALPS patients were not able to form networks of TNTs. This points toward a pivotal 
Table 1 | Overview of the different cargos found in TNT-like structures.

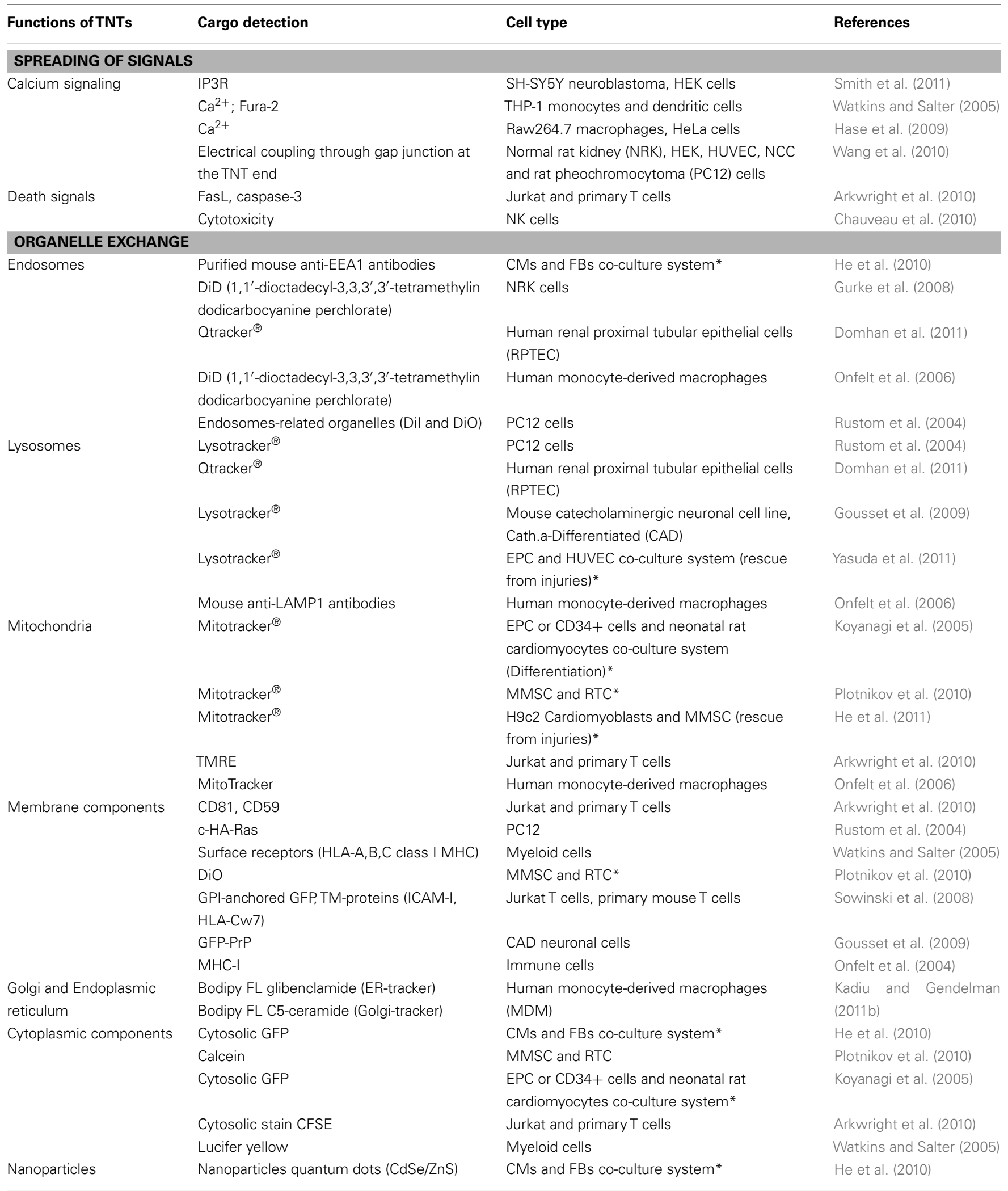


Table 1 | Continued

\begin{tabular}{|c|c|c|c|}
\hline Functions of TNTs & Cargo detection & Cell type & References \\
\hline \multicolumn{4}{|c|}{ PATHOGENS SPREADING } \\
\hline Bacteria & Mycobacterium bovis BCG & Human monocyte-derived macrophages & Onfelt et al. (2006) \\
\hline \multirow[t]{3}{*}{ Virus } & Gag and Env (antibodies), GFP-Gag & $\begin{array}{l}\text { Jurkat } T \text { cells, activated primary human or } \\
\text { primary mouse } T \text { cells }\end{array}$ & Sowinski et al. (2008) \\
\hline & HIV particles, HIV-p24 & Primary human macrophages infected by HIV & Eugenin et al. (2009) \\
\hline & Env and Gag proteins & Human monocyte-derived macrophages & $\begin{array}{l}\text { Kadiu and Gende } \\
\text { man (2011a), Kadiu an } \\
\text { Gendelman (2011b) }\end{array}$ \\
\hline \multirow[t]{2}{*}{$\begin{array}{l}\text { Proteinaceous } \\
\text { aggregates }\end{array}$} & $\operatorname{PrPSc}^{\mathrm{S}}$ & $\begin{array}{l}\text { CAD neuronal cells, GCN and DC co-culture } \\
\text { system }\end{array}$ & Gousset et al. (2009) \\
\hline & A-b fusion proteins & Astrocytes and neurons & Wang et al. (2010) \\
\hline
\end{tabular}

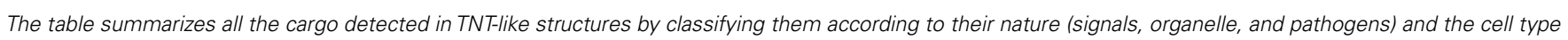
in which they were found. ${ }^{*}$ Exchange of cargos observed in co-culture of different cell type.

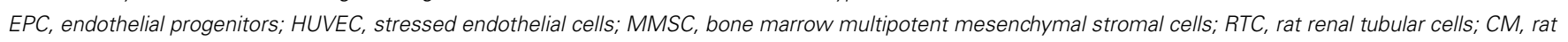
ventricular cardiomyocytes; FB, cardiofibroblasts.

role of the Fas-mediated pathway in promoting TNT formation and transfer in T cells. Additionally, transfer of both membrane (detected by CD59 and CD81 staining) and cytoplasmic components was detected in Fas-induced TNTs. Interestingly, FasL and active caspase- 3 passage from Fas-activated cells in neighboring non-activated ones was detected, thus resulting in the spreading of apoptosis through fratricide, highlighting that this might be an efficient way to shut down cellular responses (Arkwright et al., 2010). Moreover, it has been reported that FasL is upregulated in cancer cells (O'Connell et al., 1996) and this could confer a double advantage to these cells in "counterattacking" the immune system and stimulating their own proliferation. In this light, TNTs could act as conduits for diverse signals between tumor cells (for their own survival) and from tumor cells to immune cells (for death), thus leading to opposite effects.

Finally, Chauveau et al. (2010) have recently observed that also Natural Killer immune cells (NK cells) can easily form intercellular nanotubes, particularly upon activation. NK cells are important immune cells implicated in defense against a range of infections (Herberman and Ortaldo, 1981). The authors demonstrated that human primary NK cells are able to connect with different cell types by intercellular bridges and use them to mediate cytotoxicity (Table 1) and, therefore, help lyse remote target cells leading to cell death (Chauveau et al., 2010).

\section{ORGANELLE TRANSFER}

Tunneling nanotubes can in certain cases be highways for diverse organelle transfer (Table 1). Labeling with membrane-specific dyes, markers of the endo-lysosomal pathway, or other dyes specific to organelles such as mitochondria, has revealed subcellular organelles traveling between cells along these connections (Table 1). A range of cell types, including $\mathrm{T}$ cells, macrophages, NRK, stem cells, epithelial cells, myocardial cells have exhibited transfer of mitochondria (Table 1). Differentiation of embryonic endothelial progenitor cells (EPC) in myocyte-like phenotype was observed when EPC were co-cultured with neonatal rat cardiomyocytes suggesting that TNT-mediated transfer of mitochondria could have a reprogramming function in these cells (Koyanagi et al., 2005). Moreover, Spees et al. (2006) have observed the passage of mitochondria from adult non-hematopoietic stem cells (from human bone marrow hMSCs) or skin fibroblasts to A549 $\rho^{\circ}$ epithelial cells that were defective or deleted in mtDNA rescue aerobic respiration. However, the authors could only hypothesize an involvement of tubular connections between the two-cell types without demonstrating it. A closer look at some recent work involving the use of co-culture systems shows that TNTmediated mitochondrial transfer could indeed rescue injured cells for pathological conditions (Cselenyák et al., 2010). For example, Cselenyak and coworkers set up a co-culture system of H9c2 cardiomyoblasts and mesenchymal stem cells (MSC) mimicking ischemic damage in $\mathrm{H} 9 \mathrm{c} 2$ cells by using oxygen glucose deprivation (OGD). They were able to show passage of functionally active mitochondria (labeled with Mitotracker dye) in the damaged cells specifically when nanotubular connections between the cells were present (Cselenyák et al., 2010). In addition, selective bi-directional transfer of mitochondria in between connected rat ventricular cardiomyocytes (CMs) and cardiofibroblasts (FBs) was observed in tubular structures (He et al., 2011). These connections were enriched in actin and microtubules and allowed for the traffic of soluble cytosolic dyes as well, suggesting continuity between the membranes. The authors also explored a possible physiological significance of the nanotubular structures found in CMs-FBs co-culture system in vitro by culturing mouse heart tissue slices. By labeling CMs and FBs with WGA and other specific markers, the authors were able to detect thin structures between the two-cell types, reminiscent of the connections observed in vitro (He et al., 2011).

A rescue function of TNT-mediated organelle transfer might be associated with other cell types that undergo injuries as well (Table 1). Accordingly, the observation cell-to-cell contacts established between RTC and MMSC leads to the hypothesis that the exchange of cytoplasmic and organelle components could be involved in restoring functions of damaged cells following acute renal failure (Plotnikov et al., 2010). Indeed, endothelial cells 
presenting lysosomal dysfunction after exposure to AGE-modified collagen I (Yasuda et al., 2010) appeared to be rescued by transferring normal lysosomal pool from endothelial progenitors to stressed cells (Yasuda et al., 2011) This suggests a role for organelle TNT-mediated transfer in restoring functions and tissue repair, which needs to be further characterized (Table 1).

Smaller particles, named nanoparticles, have also been shown to travel within nanotubes (He et al., 2011). Particularly, Streptavidin-coated CdSe/ZnS Quantum Dots (QDs) were detected along membrane nanotubes of rat cardiac myoblast cells (H9c2) at a speed compatible with movement of DiD-labeled vesicles associated with dynein/kinesin motors walking on microtubules (Onfelt et al., 2006), thus suggesting that nanoparticles can be transported inside vesicles within these structures ( $\mathrm{He}$ et al., 2011). In fact, when WGA was used to label membrane vesicles, QDs colocalized with it inside TNTs, confirming the vesicular transport of these molecules. Moreover, like thicker TNTs described in macrophages (Onfelt et al., 2006) the nanotubes of $\mathrm{H} 9 \mathrm{c} 2$ cells contained both actin and microtubules and allowed a bi-directional transfer of membrane vesicles (He et al., 2010). Use of nanoparticles, such as QDs, is an emerging research field for diverse medical applications, such as therapies and diagnostics (Youns et al., 2011). For example, these small compounds could be used to selectively deliver drugs to cancer cells or for other infectious diseases (Singh and Nalwa, 2011). The fact that cells can establish membrane nanotubes together with the new finding that nanoparticles could pass from one cell to another by these means of communication open up new ways for diffusing small therapeutics inside target "cell communities."

\section{PATHOGEN SPREADING}

Tunneling nanotubes could be either actively hijacked from different pathogens or transport them as "Trojan horses," along the membrane or inside, leading to the spreading of infection (Table 1). Hijacking of these structures can be preceded by induction of TNT formation, thus optimizing pathogen transfer, as has been shown for HIV particles spreading, both surfing on or inside TNTs in primary macrophages (Eugenin et al., 2009). The HIV virus can use these highways to spread as an alternative to the other means already mentioned above.

Recently, a more detailed characterization of HIV-carriers mediating the transfer of the virus along TNTs bridging macrophages has been made that the authors called bridging conduits (BCs; Kadiu and Gendelman, 2011b). In this work, the authors first observed an increase in the number of connections in macrophages, as previously described (Sowinski et al., 2008). They then identified the composition of BCs by proteomic analysis following isolation from cell bodies. Although the approach used to isolate intercellular connections could not totally exclude the presence of other cellular protrusions, the work gives some insights on the possible compositions of BCs in the context of HIV spread. Indeed, they found several organelle markers including endo-lysosomal compartment (14\%), ER (9\%), and Golgi (4\%) inside BCs, the majority of which were regulators of different steps within the HIV life cycle. They were also able to confirmed by confocal microscopy that $72 \%$ of Golgi and 32\% of ER colocalize in TNTs with the viral protein Env; similar results were also obtained for the viral protein Gag, suggesting a role for these intracellular compartments in HIV intracellular trafficking (Kadiu and Gendelman, 2011a). Indeed, Golgi and ER represent sorting stations for the virus prior to reaching endosomal vesicles and before spreading. Additionally, they observed that Golgi and ER undergo morphological changes upon HIV infection (Kadiu and Gendelman, 2011a). Overall these observations shed light on a possible new role for the Golgi and ER in TNT-mediated transfer of diverse cellular components and their regulation mechanisms that need to be further investigated.

Additional observations on the trafficking of HIV have shown that HIV specifically traffics in TNTs associated with endocytic compartments and so these organelles could be responsible for viral spread between macrophages (Kadiu and Gendelman, 2011a). Moreover, the acto-myosin machinery used by the cell to move virus-containing cargoes within TNTs is 25 times faster than the surfing process seen for HIV and other retroviruses on filopodial protrusions (Sherer et al., 2007). In particular, HIV preferentially associates in TNTs with recycling endosomes and MVB (Kadiu and Gendelman, 2011a). Whether viral particles spreading in vesicles through $\mathrm{BC}$ results in a productive infection of a recipient cell and how the flow of these carriers is regulated and intersects with the intracellular pathway remain to be investigated. Comparing intra- and inter-cellular trafficking with our current knowledge in the HIV field could improve our understanding and help in characterizing intercellular spreading of other pathogens that manipulate host intracellular components for their own survival, leading to progressive loss of cellular identity.

One of the best known mechanisms of cell-to-cell spread, common in some pathogenic bacteria such as Listeria, Shigella, and Salmonella, is their ability to polymerize the host actin cytoskeleton to escape the host and keep infecting new targeted cells (Cossart and Sansonetti, 2004). While little was known about other atypical cytoplasmic bacteria spreading, recently, new "unusual" ways of bacterial spreading have been observed. For example, it has recently been shown that Cryptococcus neoformans is able to laterally transfer from an infected macrophage to an uninfected one allowing a latent persistency in the host for long periods before causing meningoencephalitis in the central nervous system (CNS; Ma et al., 2007). The authors observed an actin-dependent transfer of the bacterium in both immortalized cell lines and human primary macrophages by a mechanism not yet understood. More recently, it has been reported that the obligate intracellular bacterium Ehrlichia chaffeensis associates with filopodia of infected DH82 monocytes and increases their numbers and lengths (Thomas et al., 2010). The authors hypothesized that the transport of Ehrlichia through filopodia could be a potential mechanism for the pathogen to pass from one cell to another without contacting the extracellular environment. Another unusual way of spreading recently highlighted is the formation of an actin barrel (Hagedorn et al., 2009), the "ejectosome," induced by Mycobacterium marinum and used by it to pass within infected Dictyostelium discoideum ameba as host. This mechanism is an alternative to the formation of a protrusion containing the pathogen created by actin polymerization that is then engulfed by adjacent cells (Carlsson and Brown, 2009). Onfelt et al. (2006) have shown that M. bovis BCG or clusters of several bacteria can surf on thin 
membrane nanotubes between macrophages before being internalized by receptor-mediated endocytosis (Onfelt et al., 2006), pointing toward a possible role of these structures in bacterial infection by concentrating the pathogen on the entry site for a more efficient invasion.

Additionally, one could also envisage a role for these newly discovered highways in the spreading of some obligatory intracellular bacteria, unable to surf along TNT membranes that could use them to escape from the immune response. As already mentioned above, different sub-cellular organelles are found to shuttle in between cells by TNTs. Bacteria can use different endocytic compartments and modulate them to escape lysosomal degradation (Ham et al., 2011). In particular, vacuoles-containing bacteria deriving from fusion of the pathogen with intracellular organelles were found to be positive for several endosomal proteins (Bonazzi and Cossart, 2006). A problem for nanotubes in transporting these bigger cargoes along their tracks could be overcome by the presence of expansions along the tunnel, known as gondolas (Hurtig et al., 2010). Veranic et al. (2008) have observed that these dilatations of the membrane can move for $5-15 \mu \mathrm{m}$ with an average speed of $40 \mathrm{~nm} / \mathrm{s}$ (Veranic et al., 2008). This "pearling" phenomenon seen along some TNT structures might be due to the redistribution of lipids and cytoskeleton components localized in discrete areas and could be compatible with a vesicular transport of pathogen as well.

\section{SPREADING OF PRIONS AND PRION-LIKE NEURODEGENERATIVE DISEASES}

The mechanisms of prion spreading from the periphery to the CNS, and subsequently within the CNS, remain questionable. A number of mechanisms, such as cell-to-cell contact, exosomes, and GPI-painting, have been proposed (Baron et al., 2002; Kanu et al., 2002; Fevrier et al., 2004). We have recently demonstrated the presence of TNTs in neuronal CAD cell cultures (Gousset and Zurzolo, 2009; Table 1). In addition, we showed that these TNTs were able to transfer lysosomal organelles, the cellular GPI-anchored prion protein $\mathrm{PrP}^{\mathrm{C}}$, as well as fluorescently labeled infectious prion particles, $\mathrm{PrP}^{\mathrm{Sc}}$. Using various co-culture conditions, we demonstrated that these infectious particles were efficiently transferred to noninfected cells only in the presence of TNTs (Gousset et al., 2009).

Since the prion protein is a GPI-anchored protein, it has the possibility of traveling via TNTs either along their surface or inside the tube within vesicular structures (Figure 2 F and enlarged box). Recently we have further analyzed the presence of $\mathrm{PrP}^{\mathrm{Sc}}$ and various organelles inside TNTs. Overall, we observed that similar to what can be found in the cell body, $\mathrm{PrP}^{\mathrm{Sc}}$ travels in TNTs in early endosomes and lysosomes but it is preferentially enriched in the endosomal recycling compartment. Additionally, increasing the number of TNTs formed, by over-expression of Myosin-X, also increases the spreading of $\mathrm{PrP}^{\mathrm{Sc}}$ to non-infected cells (data not shown). These data further demonstrate how efficient these structures are in allowing the passage of infectious prions from one cell to another.

Finally, we have also demonstrated that the transfer via TNTs of infectious prion particles resulted in the transmission of infectivity to the recipient cell. This transfer was not confined to neuronal co-cultures but was also efficient between loaded bone marrow derived dendritic cells and primary neurons (Gousset et al., 2009;
Langevin et al., 2010). Thus, our studies suggested that TNTs might play a critical role in vivo in the spreading of prions within the CNS and at the periphery (Gousset and Zurzolo, 2009).

In vivo, the players involved in the spreading of prions from the gastrointestinal tract, to the lymphoid system and to the peripheral nervous system (PNS) are still unclear (Mabbott and Bruce, 2001). Dendritic cells could bring infectious prion particles from the gut to Follicular dendritic cells, and subsequently pick up prions particles from FDCs and deliver them to the PNS. Thus, analyzing the interactions between these two-cell types might reveal important clues about prion spreading in general. We have started to address these issues. Interestingly, co-culturing DCs and FDC cell lines (Nishikawa et al., 2006) we were able to detect formation of TNT-like structures between the two-cell types (data not shown).

Overall, our studies suggest that TNTs might play a pivotal role in the spreading of prion diseases. Moreover, protein aggregation represents a common neuropathological hallmark for most other neurodegenerative disorders, including Alzheimer's, Parkinson's, Huntington's diseases, and amyotrophic lateral sclerosis (ALS) and each of them is characterized by the misfolding, followed by aggregation, of a specific protein. In particular, $\beta$-amyloid $(\beta A)$ and tau for Alzheimer's, $\alpha$-synuclein ( $\alpha$-syn) for Parkinson's, huntingtin (htt) for Huntington's disease, and superoxide dismutase-1 (SOD1) for ALS. Interestingly, it has been shown that these misfolded proteins can be transmitted experimentally in animal or cellular models (Krammer et al., 2009) where they can act as "seeds" to recruit endogenous protein into aggregates (seeding process; Figure 2G) as it is the case for $\mathrm{PrP}^{\mathrm{Sc}}$ (Gousset et al., 2009; Langevin et al., 2010). For example, it has been shown that $\alpha$-syn oligomers once internalized can trigger aggregation of endogenous cytosolic $\alpha$-syn in cultured primary cortical neurons as well as in neuronal cell lines (Danzer et al., 2007, 2009). Also, extracellular aggregated tau has been shown to enter cells and transmit a misfolded state to intracellular tau (Frost et al., 2009). In this work, the authors have been able to demonstrate that exogenous tau aggregates following their uptake readily induced fibrillation in cells over-expressing a fluorescently labeled form of tau (Full-length Tau-YFP). Interestingly, the resulting aggregated form of endogenous Tau-YFP is able to seed the fibrillation of tau monomer in vitro and can transfer between cells (Frost et al., 2009). Taken together, these findings support the idea that other neurodegenerative diseases linked to protein misfolding could be considered prion-like disorders, possibly extending some features of prions to other protein pathologies (reviewed in Frost and Diamond, 2009; Brundin et al., 2010; Dunning et al., 2011; Lee et al., 2011). Furthermore, considering that these diseases follow anatomical pathways for their propagation in the brain (Brundin et al., 2010), it is tempting to speculate the possible common spreading mechanisms of different proteinaceous aggregates that might contribute to the progression of neurodegeneration (Figure 2).

As already mentioned before, transfer of prion-like aggregates between cells has been shown in in vitro cell culture models and different mechanisms of transfer have been proposed including endo/exocytosis, exosomes, trans-synaptic transmission at axonal terminals (Aguzzi and Rajendran, 2009). Consistently, $\alpha-$ syn can move between neurons in culture (Desplats et al., 2009). In this work, a co-culture system consisting of SH-SY5Y cells 


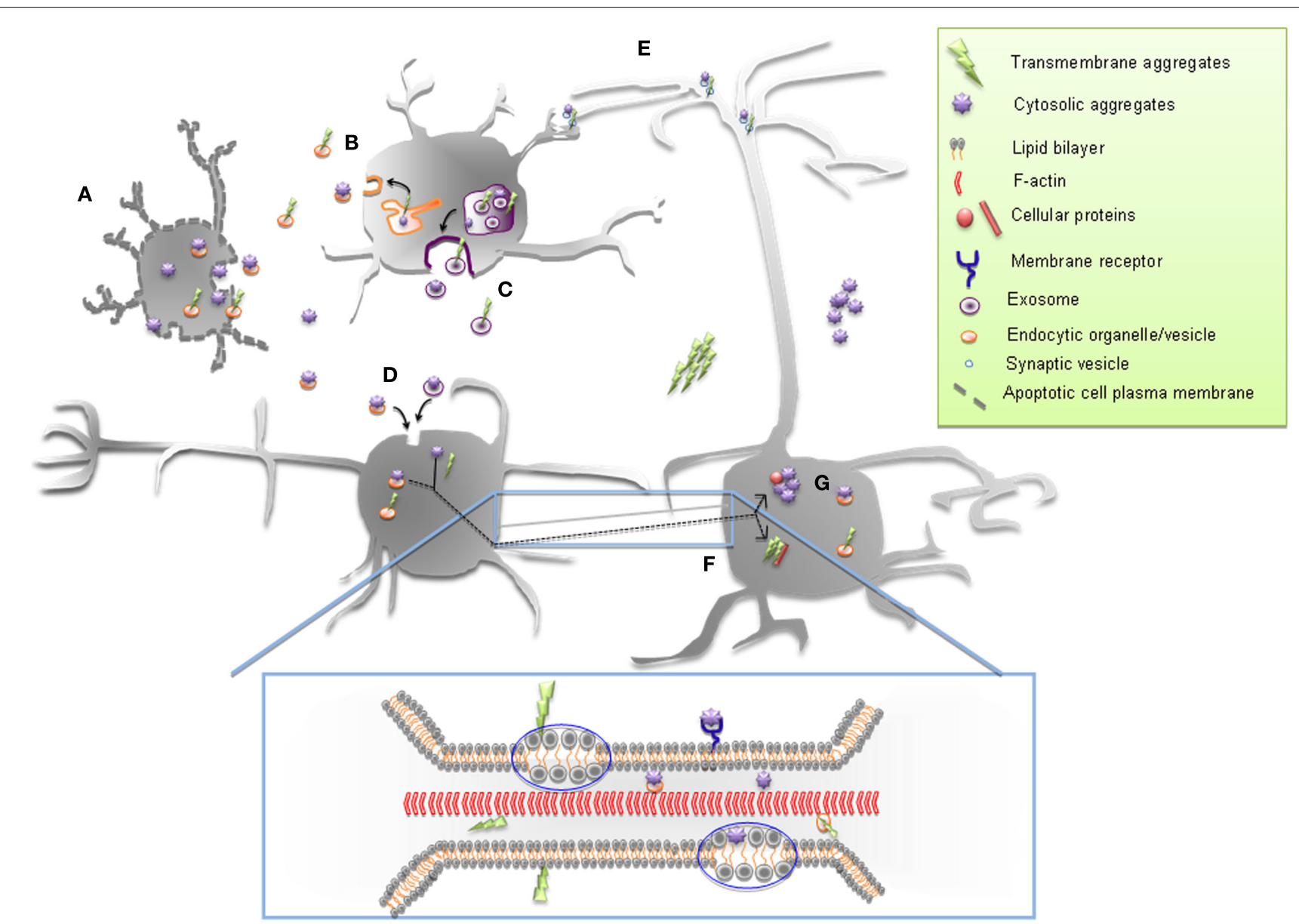

FIGURE 2 | Possible mechanisms of cell-to-cell spreading of cytosolic and transmembrane proteinaceous aggregates. Both cytosolic and transmembrane protein aggregates can be released in the extracellular space from apoptotic cell (A), by exocytosis (B) and through exosomes (C) and endocytosed by neighboring cells (D). They could also move between cells trans-synaptically (E) and through tunneling nanotubes (TNTs) (F).

TNT-mediated transfer of both types of protein aggregates (enlarged box) can occur within endocytic vesicles or as aggresomes. "Surfing" on the TNT membrane could also occur: for transmembrane aggregates through their membrane attachment and for cytosolic aggregates either within the cytosolic leaflet of the TNT or along the external leaflet in association with a membrane-receptor. Once inside the recipient cell, proteinaceous aggregates can then seed aggregation of the cytosolic or transmembrane cellular counterpart (G). over-expressing a myc-tagged version of $\alpha$-syn (donor population) and SH-SY5Y cells differently labeled (acceptor population) was established. In these conditions the detection of $\alpha$-syn in the acceptor cell population was proportional to its expression level in the donor cell population. Of interest, no membrane leakage was detected suggesting that cell-to-cell $\alpha$-syn transmission occurs without cellular membrane damage and implies viable cells (Desplats et al., 2009). More recently, it has been reported that exogenous aggregates of SOD1 deriving from highly purified recombinant SOD1 protein efficiently enter Neuro-2a neuronal (N2a) cells by macropinocytosis and rapidly escape from this compartment to reach the cytosol (Münch et al., 2011). Once there, SOD1 aggregates are able to self-propagate by converting the soluble endogenous counterpart and to spread continuously between cells (Münch et al., 2011).

Although these experiments indicate the propagation of these different proteinaceous aggregates between cells, the mechanism of transfer has not been addressed yet. Recently, Wang et al. (2011) have analyzed whether intracellular $A \beta$ particles could spread through TNTs in astrocytes and neurons. Microinjection experiments demonstrated that intracellular $A \beta$-fusion proteins were able to quickly spread from cell-to-cell via TNTs (Table 1). In addition they showed that increasing the number of TNTs between the cells by $\mathrm{H}_{2} \mathrm{O}_{2}$ treatment led to an increase in neuronal cell death in co-cultures with pEGFP-A $\beta$ over-expressing astrocytes compared to pEGFP controls (Wang et al., 2011). These data suggest that $A \beta$ particle spreading via TNTs within the cultures resulted in an increase in neuronal toxicity leading to cell death. Such observations are very similar to what we found with $\operatorname{PrP}^{\mathrm{Sc}}$ spreading and propagation in primary neurons (Gousset et al., 2009; Costanzo and Zurzolo, data not shown) and suggest that other protein aggregates like prions might use TNTs as one possible spreading mechanism (Figure 2).

Despite that, one should also take into account the different nature of the protein implicated in each neuropathology. For example, differently from $\mathrm{PrP}^{\mathrm{Sc}}$ and $\mathrm{A} \beta$, that are amyloids 
anchored to the membranes, tau, htt, SOD1, and $\alpha$-syn are cytosolic (Aguzzi and Rajendran, 2009; Münch and Bertolotti, 2011), thus raising the question of how these cytosolic aggregates might spread between cells by TNTs (Figure 2F).

For example, it has been reported that $\alpha$-syn aggregates internalization was sensitive to temperature and required dynamin-1, pointing toward a role for the endocytic pathway in its entry mechanism (Desplats et al., 2009). Similarly, internalized tau partially colocalizes with dextran, indicating also an involvement of the endocytic pathway in this process (Frost et al., 2009). Thus internalized aggregates of $\alpha$-syn and tau are likely packaged into endocytic vesicles from where they have to escape by a mechanism not yet understood in order to reach the cytosol. It might therefore be possible that endocytic vesicles could "shuttle" these aggregates in TNTs connecting cells, prior to their escape from this compartment (Figure 2B). Then once they reached the recipient cell they could be released in the cytosol where they could seed the misfolding of endogenous cytosolic proteins. On the other hand, a cytosolic passage as aggresomes through TNTs could also be envisaged since TNTs have been shown to transfer cytosolic components between connected cells (Watkins and Salter, 2005; He et al., 2010; Figure 2F and enlarged box). Interestingly $\alpha$-syn and htt can interact with acidic phospholipids enriched on the cytoplasmic membrane leaflet (Kegel et al., 2005, 2009; van Rooijen et al., 2008). Therefore a "surfing" process of the "membraneassociated" protein inside TNT membranes could also be possible (Figure 2, enlarged box).

Further investigations in this direction are intriguing and can potentially open up new ways of looking at these diseases and could potentially lead to new therapeutical approaches to selectively block misfolded protein aggregates spreading with the ultimate aim of fighting them.

\section{CONCLUDING REMARKS}

Since their discovery in 2004, an enormous amount of work has been done on the characterization of TNTs in a multitude of cell types. Here, we have reviewed recent studies and highlighted advances that have been made more specifically with respect to TNT formation, the role of specific molecules and signaling pathways, as well as their different physiological roles in the spreading of various molecules, signals, and pathogens.

\section{REFERENCES}

Abounit, S., and Zurzolo, C. (2012). Wiring through tunneling nanotubes - from electrical signals to organelle transfer. J. Cell. Sci. doi: 10.1242/jcs.083279. [Epub ahead of print].

Aguzzi, A., and Rajendran, L. (2009). The transcellular spread of cytosolic amyloids, prions, and prionoids. Neuron 64, 783-790.

Arkwright, P. D., Luchetti, F., Tour, J., Roberts, C., Ayub, R., Morales, A. P., Rodríguez, J. J., Gilmore, A., Canonico, B., Papa, S., and Esposti, M. D. (2010). Fas stimulation of T lymphocytes promotes rapid intercellular exchange of death signals via membrane nanotubes. Cell Res. 20, 72-88.

Baron, G. S., Wehrly, K., Dorward, D. W., Chesebro, B., and Caughey, B. (2002). Conversion of raft associated prion protein to the protease-resistant state requires insertion of PrPres $(\mathrm{PrPSc}$ ) into contiguous membranes. EMBO J. 21, 1031-1040.

Berg, J. S., and Cheney, R. E. (2002). Myosin-X is an unconventional myosin that undergoes intrafilopodial motility. Nat. Cell Biol. 4, 246-250.

Bonazzi, M., and Cossart, P. (2006). Bacterial entry into cells: a role for the endocytic machinery. FEBS Lett. 580, 2962-2967.

What has become evident from these studies is that long distance intercellular connections between cells are not artifacts, as they were first perceived. Indeed, they have become commonly observed features found in most cell types examined. Although discovered only recently, TNT-like structures are becoming more and more a part of mainstream cell biology. The biggest hurdle however might be the large heterogeneity that exists within these structures. This is in part due to their high dynamicity. Indeed, TNTs can form quickly and have short lifetimes. They can be induced by different signals leading to different transport mechanisms. Thus, as more molecules and signaling pathways are being described as important players in both TNT formation and/or function (Abounit and Zurzolo, 2012), it will be necessary to determine whether a general mechanism might exist for most cell types or whether each cell system might have evolved its own set of mechanisms for TNT formation, stability, and function. However, because of the disparity in the requirements of specific cytoskeleton components or specific proteins, more attention might have to be put on the role of specific lipids or lipid pathways. Indeed, while most naturally occurring nanotubes require some type of cytoskeleton components, artificially made nanotubes can be pulled from synthetic vesicles. Thus, the lipid environments and their subsequent interactions with specific proteins might bridge some of the differences observed between each cell type. For example, the determination that PI3K might play a role in TNT formation (Wang et al., 2011) suggests that phosphoinositides such as PIP2 and PIP3 might play important roles. To this aim it will be important to use biophysical approaches and model membranes to determine the role that certain lipids might play in the membrane flexibility and ability to curve. Furthermore whether common membrane domains enriched in specific lipids and proteins bring important components at the base and within TNTs for both formation and transfer needs to be analyzed.

\section{ACKNOWLEDGMENTS}

The work on TNT in Chiara Zurzolo lab is supported by Agence Nationale de Recherche [PRIONTRAF ANR-09-BLAN-0122-01, and DISCover, 2009 NEUR 00203]; the European Union FP7 [grant number 222887], and by Pasteur-Weizmann Foundation (2010-2012).

Brundin, P., Melki, R., and Kopito, R. (2010). Prion-like transmission of protein aggregates in neurodegenerative diseases. Nat. Rev. Mol. Cell Biol. 11, 301-307.

Bukoreshtliev, N. V., Wang, X., Hodneland, E., Gurke, S., Barroso, J. F. V. and Gerdes, H.-H. (2009). Selective block of tunneling nanotube (TNT) formation inhibits intercellular organelle transfer between PC12 cells. FEBS Lett. 583, 1481-1488.

Carlsson, F., and Brown, E. J. (2009). Cell biology. The art of making an exit. Science 323, 1678-1679.

Chauveau, A., Aucher, A., Eissmann, P., Vivier, E., and Davis, D. M. (2010). Membrane nanotubes facilitate long-distance interactions between natural killer cells and target cells. Proc. Natl. Acad. Sci. U.S.A. 107, 5545-5550.

Chinnery, H. R., Pearlman, E., and McMenamin, P. G. (2008). Cutting edge: membrane nanotubes in vivo: a feature of MHC class II+ cells in the mouse cornea. J. Immunol. 180, 5779-5783.

Connors, B. W., and Long, M. A. (2004). Electrical synapses in the mammalian brain. Annu. Rev. Neurosci. 27, 393-418.

Cossart, P., and Sansonetti, P. J. (2004). Bacterial invasion: the paradigms of enteroinvasive pathogens. Science $304,242-248$. 
Cselenyák, A., Pankotai, E., Horváth, E. M., Kiss, L., and Lacza, Z. (2010). Mesenchymal stem cells rescue cardiomyoblasts from cell death in an in vitro ischemia model via direct cell-to-cell connections. BMC Cell Biol. 11, 29. doi:10.1186/1471-2121-11-29

Danzer, K. M., Haasen, D., Karow, A. R., Moussaud, S., Habeck, M., Giese, A., Kretzschmar, H., Hengerer, B., and Kostka, M. (2007). Different species of alpha-synuclein oligomers induce calcium influx and seeding. $J$. Neurosci. 27, 9220-9232.

Danzer, K. M., Krebs, S. K., Wolff, M., Birk, G., and Hengerer, B. (2009). Seeding induced by alphasynuclein oligomers provides evidence for spreading of alphasynuclein pathology. J. Neurochem. 111, 192-203.

Demontis, F., and Dahmann, C. (2007). Apical and lateral cell protrusions interconnect epithelial cells in live Drosophila wing imaginal discs. Dev. Dyn. 236, 3408-3418.

Desplats, P., Lee, H.-J., Bae, E.-J., Patrick, C., Rockenstein, E., Crews, L., Spencer, B., Masliah, E., and Lee, S.-J. (2009). Inclusion formation and neuronal cell death through neuron-to-neuron transmission of alpha-synuclein. Proc. Natl. Acad. Sci. U.S.A. 106, 13010-13015.

Domhan, S., Ma, L., Tai, A., Anaya, Z., Beheshti, A., Zeier, M., Hlatky, L., and Abdollahi, A. (2011). Intercellular communication by exchange of cytoplasmic material via tunneling nano-tube like structures in primary human renal epithelial cells. PLoS ONE 6, e21283. doi: 10.1371/journal.pone.0021283

Dubey, G. P., and Ben-Yehuda, S. (2011). Intercellular nanotubes mediate bacterial communication. Cell 144, 590-600.

Dunning, C. J. R., Reyes, J. F., Steiner, J. A., and Brundin, P. (2011). Can Parkinson's disease pathology be propagated from one neuron to another? Prog. Neurobiol. PMID:22115849. [Epub ahead of print].

Dustin, M. L., Chakraborty, A. K., and Shaw, A. S. (2010). Understanding the structure and function of the immunological synapse. Cold Spring Harb. Perspect. Biol. 2, a002311.

Eugenin, E. A., Gaskill, P. J., and Berman, J. W. (2009). Tunneling nanotubes (TNT) are induced by HIV-infection of macrophages: a potential mechanism for intercellular HIV trafficking. Cell. Immunol. 254, 142-148.

Favoreel, H. W., Van Minnebruggen, G. Adriaensen, D., and Nauwynck, H. J.
(2005). Cytoskeletal rearrangements and cell extensions induced by the US3 kinase of an alphaherpesvirus are associated with enhanced spread. Proc. Natl. Acad. Sci. U.S.A. 102, 8990-8995

Fevrier, B., Vilette, D., Archer, F., Loew, D., Faigle, W., Vidal, M., Laude, H., and Raposo, G. (2004). Cells release prions in association with exosomes. Proc. Natl. Acad. Sci. U.S.A. 101, 9683-9688.

Frost, B., and Diamond, M. I. (2009). The expanding realm of prion phenomena in neurodegenerative disease. Prion 3, 74-77.

Frost, B., Jacks, R. L., and Diamond, M. I. (2009). Propagation of tau misfolding from the outside to the inside of a cell. J. Biol. Chem. 284, 12845-12852.

Gerdes, H.-H., Bukoreshtliev, N. V., and Barroso, J. F. V. (2007). Tunneling nanotubes: a new route for the exchange of components between animal cells. FEBS Lett. 581, 2194-2201.

Gousset, K., Schiff, E., Langevin, C., Marijanovic, Z., Caputo, A., Browman, D. T., Chenouard, N., de Chaumont, F., Martino, A., Enninga, J., Olivo-Marin, J.-C., Männel, D., and Zurzolo, C. (2009). Prions hijack tunnelling nanotubes for intercellular spread. Nat. Cell Biol. 11, 328-336.

Gousset, K., and Zurzolo, C. (2009). Tunnelling nanotubes: a highway for prion spreading? Prion 3, 94-98.

Green, D. R., Droin, N., and Pinkoski, M. (2003). Activation-induced cell death in T cells. Immunol. Rev. 193, 70-81.

Gurke, S., Barroso, J. F. V., and Gerdes, H.-H. (2008). The art of cellular communication: tunneling nanotubes bridge the divide. Histochem. Cell Biol. 129, 539-550.

Hagedorn, M., Rohde, K. H., Russell, D. G., and Soldati, T. (2009). Infection by tubercular mycobacteria is spread by nonlytic ejection from their amoeba hosts. Science 323, 1729-1733.

Ham, H., Sreelatha, A., and Orth, K. (2011). Manipulation of host membranes by bacterial effectors. Nat. Rev. Microbiol. 9, 635-646.

Hase, K., Kimura, S., Takatsu, H., Ohmae, M., Kawano, S., Kitamura, H., Ito, M., Watarai, H., Hazelett, C. C., Yeaman, C., and Ohno, H. (2009). M-Sec promotes membrane nanotube formation by interacting with Ral and the exocyst complex. Nat. Cell Biol. 11, 1427-1432.

He, K., Luo, W., Zhang, Y., Liu, F., Liu, D., Xu, L., Qin, L., Xiong, C., Lu, Z., Fang, X., and Zhang,
Y. (2010). Intercellular transportation of quantum dots mediated by membrane nanotubes. ACS Nano 4, 3015-3022.

He, K., Shi, X., Zhang, X., Dang, S., Ma, X., Liu, F., Xu, M., Lv, Z., Han, D., Fang, X., and Zhang, Y. (2011). Long-distance intercellular connectivity between cardiomyocytes and cardiofibroblasts mediated by membrane nanotubes. Cardiovasc. Res. 92, 39-47.

Herberman, R. B., and Ortaldo, J. R. (1981). Natural killer cells: their roles in defenses against disease. Science 214, 24-30.

Hurtig, J., Chiu, D. T., and Onfelt, B. (2010). Intercellular nanotubes: insights from imaging studies and beyond. Wiley Interdiscip. Rev. Nanomed. Nanobiotechnol. 2, 260-276.

Jolly, C., and Sattentau, Q. J. (2004). Retroviral spread by induction of virological synapses. Traffic 5, 643-650.

Kadiu, I., and Gendelman, H. E. (2011a). Macrophage bridging conduit trafficking of HIV-1 through the endoplasmic reticulum and Golgi network. J. Proteome Res. 10, 3225-3238.

Kadiu, I., and Gendelman, H. E. (2011b). Human immunodeficiency virus type 1 endocytic trafficking through macrophage bridging conduits facilitates spread of infection. J. Neuroimmune Pharmacol. 6 , 658-675.

Kanu, N., Imokawa, Y., Drechsel, D. N., Williamson, R. A., Birkett, C. R., Bostock, C. J., and Brockes, J. P. (2002). Transfer of scrapie prion infectivity by cell contact in culture. Curr. Biol. 12, 523-530.

Kegel, K. B., Sapp, E., Yoder, J., Cuiffo, B., Sobin, L., Kim, Y. J., Qin, Z.-H., Hayden, M. R., Aronin, N., Scott, D. L., Isenberg, G., Goldmann, W. H., and DiFiglia, M. (2005). Huntingtin associates with acidic phospholipids at the plasma membrane. J. Biol. Chem. 280, 36464-36473.

Kegel, K. B., Schewkunow, V., Sapp, E., Masso, N., Wanker, E. E., DiFiglia, M., and Goldmann, W. H. (2009). Polyglutamine expansion in huntingtin increases its insertion into lipid bilayers. Biochem. Biophys. Res. Commun. 387, 472-475.

Koyanagi, M., Brandes, R. P., Haendeler, J., Zeiher, A. M., and Dimmeler, S. (2005). Cell-to-cell connection of endothelial progenitor cells with cardiac myocytes by nanotubes. Circ. Res. 96, 1039-1041.

Krammer, C., Schätzl, H. M., and Vorberg, I. (2009). Prion-like propagation of cytosolic protein aggregates: insights from cell culture models. Prion 3, 206-212.

La Boissière, S., Izeta, A., Malcomber, S., and O'Hare, P. (2004). Compartmentalization of VP16 in cells infected with recombinant herpes simplex virus expressing VP16green fluorescent protein fusion proteins. J. Virol. 78, 8002-8014.

Langevin, C., Gousset, K., Costanzo, M. Richard Le Goff, O., and Zurzolo, C. (2010). Characterization of the role of dendritic cells in prion transfer to primary neurons. Biochem. J. 431, 189-198.

Lee, S.-J., Lim, H.-S., Masliah, E., and Lee, H.-J. (2011). Protein aggregate spreading in neurodegenerative diseases: problems and perspectives. Neurosci. Res. 70, 339-348.

Lehmann, M. J., Sherer, N. M., Marks, C. B., Pypaert, M., and Mothes, W. (2005). Actin- and myosin-driven movement of viruses along filopodia precedes their entry into cells. $J$. Cell Biol. 170, 317-325.

Lucas, W. J., Ham, B.-K., and Kim, J.Y. (2009). Plasmodesmata-bridging the gap between neighboring plant cells. Trends Cell Biol. 19, 495-503.

Ma, H., Croudace, J. E., Lammas, D. A., and May, R. C. (2007). Direct cell-to-cell spread of a pathogenic yeast. BMC Immunol. 8, 15. doi:10.1186/1471-2172-8-15

Mabbott, N. A., and Bruce, M. E. (2001). The immunobiology of TSE diseases. J. Gen. Virol. 82, 2307-2318.

Maeda, S., and Tsukihara, T. (2011). Structure of the gap junction channel and its implications for its biological functions. Cell. Mol. Life Sci. $68,1115-1129$.

Martin, D. A., Zheng, L., Siegel, R. M., Huang, B., Fisher, G. H., Wang, J., Jackson, C. E., Puck, J. M., Dale, J., Straus, S. E., Peter, M. E., Krammer, P. H., Fesik, S., and Lenardo, M. J. (1999). Defective CD95/APO$1 /$ Fas signal complex formation in the human autoimmune lymphoproliferative syndrome, type Ia. Proc. Natl. Acad. Sci. U.S.A. 96, 4552-4557.

Miller, J., Fraser, S. E., and McClay, D. (1995). Dynamics of thin filopodia during sea urchin gastrulation. Development 121, 2501-2511.

Mothes, W., Sherer, N. M., Jin, J., and Zhong, P. (2010). Virus cellto-cell transmission. J. Virol. 84 8360-8368.

Münch, C., and Bertolotti, A. (2011). Self-propagation and transmission of misfolded mutant SOD1: prion or prion-like phenomenon? Cell Cycle $10,1711$. 
Münch, C., O'Brien, J., and Bertolotti, A. (2011). Prion-like propagation of mutant superoxide dismutase1 misfolding in neuronal cells. Proc. Natl. Acad. Sci. U.S.A. 108, 3548-3553.

Nishikawa, Y., Hikida, M., Magari, M., Kanayama, N., Mori, M., Kitamura, H., Kurosaki, T., and Ohmori, H. (2006). Establishment of lymphotoxin beta receptor signalingdependent cell lines with follicular dendritic cell phenotypes from mouse lymph nodes. J. Immunol. 177, 5204-5214.

O'Connell, J., O'Sullivan, G. C., Collins, J. K., and Shanahan, F. (1996). The Fas counterattack: Fas-mediated T cell killing by colon cancer cells expressing Fas ligand. J. Exp. Med. 184, 1075-1082.

Onfelt, B., Nedvetzki, S., Benninger, R. K. P., Purbhoo, M. A., Sowinski, S., Hume, A. N., Seabra, M. C., Neil, M. A. A., French, P. M. W., and Davis, D. M. (2006). Structurally distinct membrane nanotubes between human macrophages support longdistance vesicular traffic or surfing of bacteria. J. Immunol. 177, 8476-8483.

Onfelt, B., Nedvetzki, S., Yanagi, K., and Davis, D. M. (2004). Cutting edge: membrane nanotubes connect immune cells. J. Immunol. 173, 1511-1513.

Plotnikov, E. Y., Khryapenkova, T. G., Galkina, S. I., Sukhikh, G. T., and Zorov, D. B. (2010). Cytoplasm and organelle transfer between mesenchymal multipotent stromal cells and renal tubular cells in co-culture. Exp. Cell Res. 316, 2447-2455.

Ramírez-Weber, F. A., and Kornberg, T. B. (1999). Cytonemes: cellular processes that project to the principal signaling center in Drosophila imaginal discs. Cell 97, 599-607.

Rechavi, O., Goldstein, I., Vernitsky, H., Rotblat, B., and Kloog, Y. (2007). Intercellular transfer of oncogenic H-Ras at the immunological synapse. PLoS ONE 2, e1204. doi:10.1371/journal.pone.0001204
Rupp, I., Sologub, L., Williamson, K. C., Scheuermayer, M., Reininger, L., Doerig, C., Eksi, S., Kombila, D. U., Frank, M., and Pradel, G. (2011). Malaria parasites form filamentous cell-to-cell connections during reproduction in the mosquito midgut. Cell Res. 21, 683-696.

Rustom, A. (2009). Hen or egg? Some thoughts on tunneling nanotubes. Ann. N. Y. Acad. Sci. 1178, 129-136.

Rustom, A., Saffrich, R., Markovic, I., Walther, P., and Gerdes, H.H. (2004). Nanotubular highways for intercellular organelle transport. Science 303, 1007-1010.

Schwann, T., and Schleyden, M. J. (1847). Microscopical Researches into the Accordance in the Structure and Growth of Animals and Plants. Printed for the Sydenham Society, London.

Sherer, N. M., Lehmann, M. J., Jimenez-Soto, L. F., Horensavitz, C., and Pypaert Mothes, W. (2007). Retroviruses can establish filopodial bridges for efficient cell-tocell transmission. Nat. Cell Biol. 9, 310-315.

Singh, R., and Nalwa, H. S. (2011). Medical applications of nanoparticles in biological imaging, cell labeling, antimicrobial agents, and anticancer nanodrugs. J. Biomed. Nanotechnol. 7, 489-503.

Smith, I. F., Shuai, J., and Parker, I. (2011). Active generation and propagation of $\mathrm{Ca} 2+$ signals within tunneling membrane nanotubes. Biophys. J. 100, L37-L39.

Sowinski, S., Jolly, C., Berninghausen, O., Purbhoo, M. A., Chauveau, A., Köhler, K., Oddos, S., Eissmann, P., Brodsky, F. M., Hopkins, C., Onfelt, B., Sattentau, Q., and Davis, D. M. (2008). Membrane nanotubes physically connect $\mathrm{T}$ cells over long distances presenting a novel route for HIV-1 transmission. Nat. Cell Biol. $10,211-219$.

Spees, J. L., Olson, S. D., Whitney, M. J., and Prockop, D. J. (2006). Mitochondrial transfer between cells can rescue aerobic respiration. Proc. Natl. Acad. Sci. U.S.A. 103, 1283-1288.

Süudhof, T. C. (2008). Neurotransmitter release. Handb. Exp. Pharmacol. 184, 1-21.

Tarakanov, A. O., and Goncharova, L B. (2009). Cell-cell nanotubes. Commun. Integr. Biol. 2, 359-361.

Thomas, S., Popov, V. L., and Walker, D. H. (2010). Exit mechanisms of the intracellular bacterium Ehrlichia. PLoS ONE 5, e15775. doi:10.1371/journal.pone.0015775

van Rooijen, B. D., Claessens, M. M. A. E., and Subramaniam, V. (2008). Membrane binding of oligomeric alpha-synuclein depends on bilayer charge and packing. FEBS Lett. 582, 3788-3792.

Veranic, P., Lokar, M., Schütz, G. J., Weghuber, J., Wieser, S., Hägerstrand, H., Kralj-Iglic, V., and Iglic, A. (2008). Different types of cell-tocell connections mediated by nanotubular structures. Biophys. J. 95, 4416-4425.

Wang, X., and Gerdes, H.-H. (2011). Long-distance electrical coupling via tunneling nanotubes. Biochim. Biophys. Acta. PMID:21930113. [Epub ahead of print]

Wang, X., Veruki, M. L., Bukoreshtliev, N. V., Hartveit, E., and Gerdes, H.H. (2010). Animal cells connected by nanotubes can be electrically coupled through interposed gapjunction channels. Proc. Natl. Acad. Sci. U.S.A. 107, 17194-17199.

Wang, Y., Cui, J., Sun, X., and Zhang, Y. (2011). Tunneling-nanotube development in astrocytes depends on p53 activation. Cell Death Differ. 18 732-742.

Watkins, S. C., and Salter, R. D. (2005). Functional connectivity between immune cells mediated by tunneling nanotubules. Immunity 23 309-318.

Wolpert, L., and Gustafson, T. (1961) Studies on the cellular basis of morphogenesis of the sea urchin embryo. The formation of the blastula. Exp. Cell Res. 25, 374-382.
Yasuda, K., Khandare, A., Burianovskyy, L., Maruyama, S., Zhang, F., Nasjletti, A., and Goligorsky, M. S. (2011). Tunneling nanotubes mediate rescue of prematurely senescent endothelial cells by endothelial progenitors: exchange of lysosomal pool. Aging (Albany N. Y.) 3, 597-608.

Yasuda, K., Park, H.-C., Ratliff, B., Addabbo, F., Hatzopoulos, A. K., Chander, P., and Goligorsky, M. S. (2010). Adriamycin nephropathy. Am. J. Pathol. 176, 1685-1695.

Youns, M., Hoheisel, J. D., and Efferth, T. (2011). Therapeutic and diagnostic applications of nanoparticles. Curr. Drug Targets 12, 357-365.

Zani, B. G., and Edelman, E. R. (2010). Cellular bridges. Commun. Integr. Biol. 3, 215-220.

Zani, B. G., Indolfi, L., and Edelman, E. R. (2010). Tubular bridges for bronchial epithelial cell migration and communication. PLoS ONE 5, e8930. doi:10.1371/journal.pone. 0008930

Conflict of Interest Statement: The authors declare that the research was conducted in the absence of any commercial or financial relationships that could be construed as a potential conflict of interest.

Received: 21 December 2011; paper pending published: 17 January 2012; accepted: 13 March 2012; published online: 10 April 2012.

Citation: Marzo L, Gousset K and Zurzolo C (2012) Multifaceted roles of tunneling nanotubes in intercellular communication. Front. Physio. 3:72. doi: 10.3389/fphys.2012.00072

This article was submitted to Frontiers in Membrane Physiology and Biophysics, a specialty of Frontiers in Physiology. Copyright (c) 2012 Marzo, Gousset and Zurzolo. This is an open-access article distributed under the terms of the Creative Commons Attribution Non Commercial License, which permits noncommercial use, distribution, and reproduction in other forums, provided the original authors and source are credited. 